\title{
Numerical Simulations of the Flow Field Ahead of an Accelerating Flame in an Obstructed Channel
}

\author{
C.Johansen**and G. Ciccarelli* ${ }^{*}$ \\ Department of Mechanical and Materials Engineering, Queen's University, Kingston, \\ Canada
}

March 23, 2010

\begin{abstract}
The development of the unburned gas flow field ahead of a flame front in an obstructed channel was investigated using large eddy simulation (LES). The standard Smagorinsky-Lilly and dynamic Smagorinsky-Lilly subgrid models were used in these simulations. The geometry is essentially twodimensional. The fence-type obstacles were placed on the top and bottom surfaces of a square crosssection channel, equally spaced along the channel length at the channel height. The laminar rollup of a vortex downstream of each obstacle, transition to turbulence, and growth of a recirculation zone between consecutive obstacles were observed in the simulations. By restricting the simulations to the early stages of the flame acceleration and by varying the domain width and domain length, the three-dimensionality of the vortex rollup process was investigated. It was found that initially the rollup process was twodimensional and unaffected by the domain length and width. As the recirculation zone grew to fill the streamwise gap between obstacles, the length and width of the computational domain started to affect the simulation results. Three-dimensional flow structures formed within the shear layer, which was generated near the obstacle tips, and the core flow was affected by large-scale turbulence. The simulation predictions were compared to experimental schlieren images of the convection of helium tracer. The development of recirculation zones resulted in the formation of contraction and expansion regions near the obstacles, which significantly affected the centerline gas velocity. Oscillations in the centerline unburned gas velocity were found to be the dominate cause for the experimentally observed early flame-tip velocity oscillations. At later simulation times, regular oscillations in the unburned streamwise gas velocity were not observed, which is contrary to the experimental evidence. This suggests that fluctuations in the burning rate might be the source of the late flame-tip velocity oscillations. The effect of obstacle blockage ratio (BR) on the development of the unburned gas flow field was also investigated by varying the obstacle height. Simulation predictions show favorable agreement to the experimental results and indicate that turbulence production increases with increasing obstacle BR.
\end{abstract}

\section{Nomenclature}

\section{Abbreviations}

\begin{tabular}{l|l} 
BR & blockage ratio \\
CFL & Courant-Friedrich-Levi stability limit (non-dimensional time) \\
LES & large eddy simulation \\
SIMPLE & $\begin{array}{l}\text { semi-implicit method for pressure-linked equations } \\
\text { UDF }\end{array}$ \\
user defined function
\end{tabular}

\footnotetext{
** Corresponding author. Email: johansen@ucalgary.ca (Now at the University of Calgary)

$\dagger^{*}$ Co-author. Email: ciccarel@me.queensu.ca
} 


\section{English}

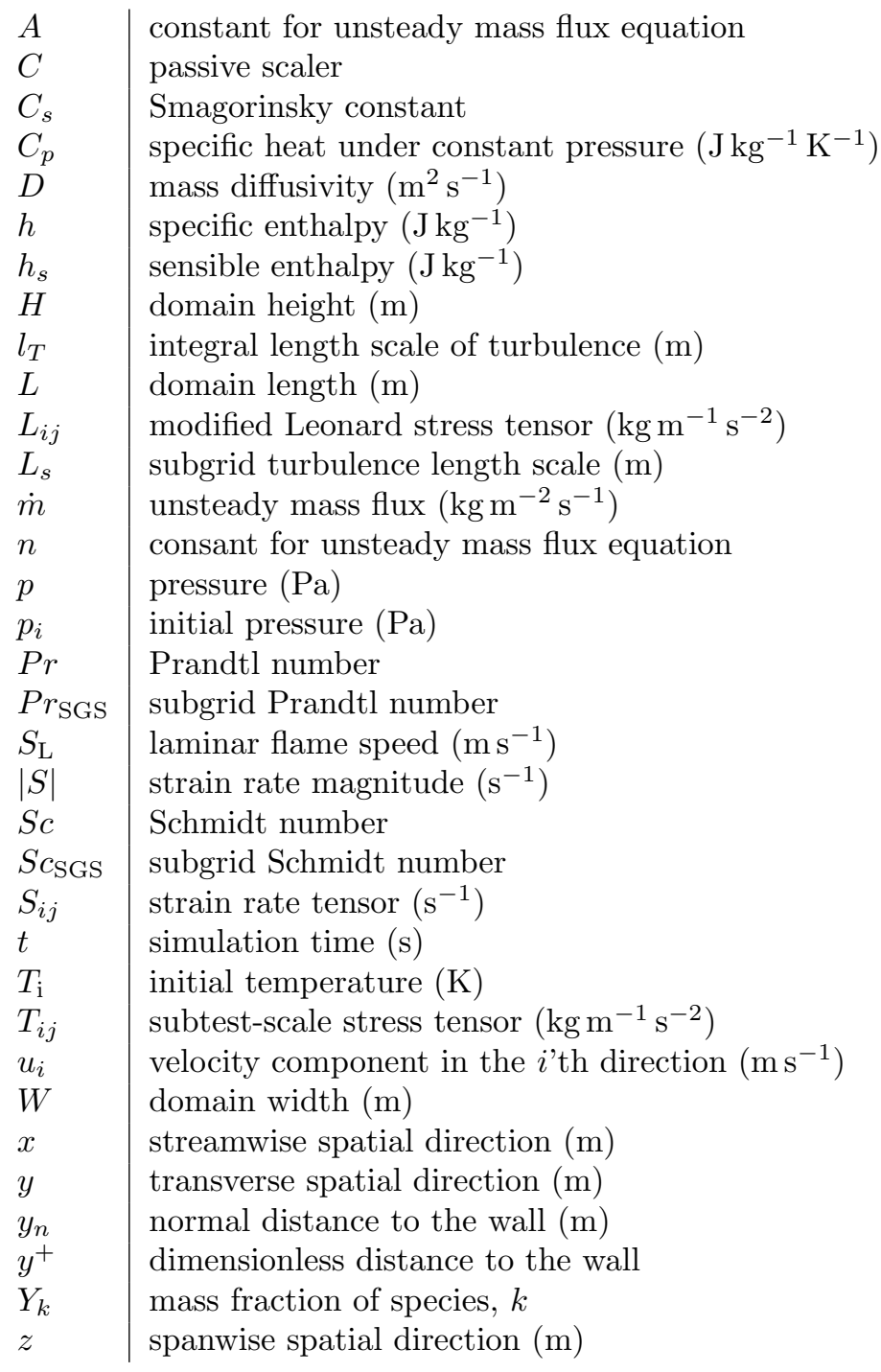

\section{Greek}

\begin{tabular}{l|l}
$\Delta$ & grid node spacing $(\mathrm{m})$ \\
$\tilde{\vartheta}$ & favre-averaged variable \\
$\bar{\vartheta}$ & filtered variable \\
$\rho$ & density $\left(\mathrm{kg} \mathrm{m}^{-3}\right)$ \\
$\kappa$ & von Kármán constant $(=0.41)$ \\
$\lambda$ & thermal conductivity $\left(\mathrm{W} \mathrm{m}^{-1} \mathrm{~K}^{-1}\right)$ \\
$\mu$ & dynamic viscosity $\left(\mathrm{kg} \mathrm{m}^{-1} \mathrm{~s}^{-1}\right)$ \\
$\mu_{\mathrm{SGS}}$ & subgrid viscosity $\left(\mathrm{kg} \mathrm{m}^{-1} \mathrm{~s}^{-1}\right)$ \\
$\sigma_{i j}$ & viscous stress tensor $\left(\mathrm{kg} \mathrm{m}^{-1} \mathrm{~s}^{-2}\right)$ \\
$\tau_{i j}$ & subgrid stress tensor $\left(\mathrm{kg} \mathrm{m}^{-1} \mathrm{~s}^{-2}\right)$ \\
$\tau_{\mathrm{w}}$ & wall shear stress $\left(\mathrm{kg} \mathrm{m}^{-1} \mathrm{~s}^{-2}\right)$ \\
$\omega$ & vorticity $\left(\mathrm{s}^{-1}\right)$
\end{tabular}




\section{Subscripts}

\begin{tabular}{l|l}
$\mathrm{SGS}$ & subgrid scale \\
$i, j, k$ & index counts in the three grid directions; $x, y, z$ components of a quantity
\end{tabular}

\section{Introduction}

The study of flame acceleration in an obstructed channel has applications ranging from explosion safety to the development of pulse detonation engines (PDEs) [1,2]. Under certain conditions, flame acceleration can lead to deflagration-to-detonation transition (DDT). A detonation wave is a supersonic mode of combustion which produces a large dynamic pressure. From the onset of spark ignition at the closed end of a channel, the development of the unburned gas flow field has a large influence on the rate of flame acceleration prior to DDT [3]. The unburned gas flow field is driven by the expansion of combustion products behind the flame front and becomes distorted due to the geometry of the confinement ahead of the flame front. In the present study, obstacles mounted on the top and bottom channel surfaces are responsible for distorting the unburned gas flow field. The generation of a shear layer from the obstacle tips results in the production of turbulence, which increases the total transport of mass, momentum, and energy in the flow. Large-scale unburned gas flow distortions cause the flame to "fold" thereby increasing the flame area. As the flame propagates into regions characterized by high levels of turbulence, small-scale wrinkling further increases the total flame area. An increase in the volumetric burning rate resulting from flame area production causes the expansion rate of the combustion products to increase thereby increasing the unburned gas velocity. A feedback loop is formed between the volumetric burning rate and the unburned gas velocity, which leads to flame acceleration. At the later stages of flame acceleration prior to DDT, shock-flame interactions become the dominant mechanism responsible for flame acceleration [1].

In this study, however, attention is focused on the initial stages of flame acceleration where shock-flame interactions and other compressibility effects are not important. Understanding the initial stages of flame acceleration is essential as they are responsible for creating the conditions required for DDT. Due to the relatively low flame speeds, the time scales associated with the initial stage of flame acceleration are much longer than the time scales associated with the later stages of flame acceleration. In the context of PDEs, which must operate at high frequencies to provide adequate thrust, effort spent minimizing the time duration of the initial stages of flame acceleration can greatly improve the performance of the engine [4]. In the context of explosion safety, inhibiting the rate of flame area production during the inital stages of flame acceleration can reduce the total overpressure and perhaps prevent a detonation initiation event [5].

Recent numerical simulations of flame propagation in obstructed channels have given insight into the mechanisms responsible for flame acceleration. Gamezo et al. [6] simulated flame acceleration in an obstructed channel from spark ignition up to the initiation and propagation of a detonation wave. Simulations were completed on a two-dimensional, adaptive grid, which allowed the hydrogen-air flame thickness to be resolved with approximately 18 nodes. The simulation used single step chemistry modeled with Arrhenius kinetics and the grid was refined dynamically at each time step based on gradients of density, pressure, velocity, and mixture composition. Since the main focus of the study was to predict the DDT event, which was due to shock reflection from an obstacle surface, the turbulence field was spatially under-resolved and no turbulence model was used. Bychkov et al. [7] performed simulations of the initial stages of flame acceleration in an obstructed channel prior to DDT for the purpose of developing an analytical model to predict changes in the flame speed. Similarly, the turbulence field was under-resolved and no turbulence model was used. Recent simulations of flame propagation past obstructions using large eddy simulation to treat the turbulence field have been undertaken $[8,9]$. These studies were mainly focused on the development of the flame surface and comparing the flame-tip speed to experimental data. A simulation which is entirely focused on predicting the development of the unburned gas flow field ahead of the flame, and which can be directly validated with experimental data, is required.

A novel experimental technique was developed by Johansen and Ciccarelli [10] to visualize the development of the unburned gas flow field by injecting a small amount of helium tracer gas ahead of the flame front. All of the experiments were carried out using stoichiometric methane-air at an initial pressure and temperature of $p_{i}=47 \mathrm{kPa}$ and $T_{i}=293 \mathrm{~K}$, respectively. The channel had a total length of $2.44 \mathrm{~m}$ and a 
square cross-section of $7.6 \mathrm{~cm} \times 7.6 \mathrm{~cm}$. Obstacles of varying blockage ratios $(\mathrm{BR}=0.33,0.5,0.67)$ were distributed along the channel top and bottom surfaces spaced at a distance equivalent to the channel height. Figure 2 illustrates how the visualization technique was used in a test with 0.5 BR obstacles. Helium tracer gas was injected $38.1 \mathrm{~cm}$ downstream of the ignition point transversely via a port through the top surface of the channel between obstacles prior to ignition. After ignition, the expansion of combustion products induced a flow in the unburned gas, displacing the helium/methane-air interface, which was visible in the schlieren images. By observing the evolution of these interfaces, several phenomena were observed. Initially, the unburned gas flow separated and formed a pair of laminar vortices downstream of each obstacle with well defined spirals in their cores. The flow at the upstream free-edge of the obstacle then detached and formed an unstable shear layer that disrupted the roll-up of each laminar vortex. The new vortices that formed were turbulent and no discernible spiral structure was visible in the cores. The shear layer grew in length, filling the gap between consecutive obstacles and separated the recirculation zone from the laminar core flow. Experimentally, it was found that the shape of the flame surface was very similar to the shape of the helium/methane-air interface at a given channel position. In addition, it was found that the obstacle blockage ratio (BR) played an important role in determining the initial rate of flame acceleration. In experiments with high BR obstacles, the flame surface was not immediately able to penetrate the shear layer, which formed from the obstacle free-edge, and propagate to the channel top and bottom surfaces. This caused the flame to become stretched over multiple obstacles. A narrower core flow at the channel centerline in combination with a stretched flame surface resulted in a high rate of flame acceleration compared to tests with lower BR obstacles. As shown in figure 9, the obstacle BR has a large effect on the magnitude of flame-tip velocity oscillations. These oscillations are due to the acceleration and deceleration of the unburned gas as it flows through the openings between obstacles. The amplitude of the oscillations was more significant in tests with the higher BR obstacles because of the more severe flow contraction.

Although this visualization technique gave insight into the unsteady development of the unburned gas flow field, the schlieren images were effectively two-dimensional maps that integrate all of the density gradients across the width of the channel. In addition, for a given frame rate of 3000 frames per second, the resolution of the images were restricted to $1024 \times 256$ pixels. Therefore, numerical simulations of this unsteady flow development were undertaken to retrieve more information such as the evolution of velocity, vorticity, and pressure distributions. Distributions of the helium/methane-air interface that were obtained experimentally were used to validate the numerical model. The importance of three-dimensional effects on the development of the core flow and of the shear layers was investigated. The extent to which the core flow centerline velocity was affected by the development of the recirculation zones was also studied. Large oscillations that occur in the centerline unburned gas velocity are expected to be largely responsible for the flame-tip velocity oscillations that are observed experimentally.

\section{Simulation Setup}

In these simulations, a low-Mach number formulation of the compressible filtered governing equations were solved:

$$
\begin{gathered}
\frac{\partial \bar{\rho}}{\partial t}+\frac{\partial \bar{\rho} \tilde{u}_{i}}{\partial x_{i}}=0 \\
\frac{\partial \bar{\rho} \tilde{u}_{i}}{\partial t}+\frac{\partial \bar{\rho} \tilde{u}_{i} \tilde{u}_{j}}{\partial x_{i}}+\frac{\partial \bar{p}}{\partial x_{i}}-\frac{\partial \tilde{\sigma}_{i j}}{\partial x_{j}}=-\frac{\partial}{\partial x_{j}} \underbrace{\left[\bar{\rho}\left(\widetilde{u_{i} u_{j}}-\tilde{u}_{i} \tilde{u}_{j}\right)\right.}_{\text {subgrid stress }}] \\
\frac{\partial \bar{\rho} \tilde{h}_{s}}{\partial t}+\frac{\partial \bar{\rho} \tilde{u}_{i} \tilde{h}_{s}}{\partial x_{i}}-\frac{\partial \bar{p}}{\partial t}-\tilde{u}_{j} \frac{\partial \bar{p}}{\partial x_{i}}-\frac{\partial}{\partial x_{i}}\left(\lambda \frac{\partial \tilde{T}}{\partial x_{i}}\right)=-\frac{\partial}{\partial x_{j}} \underbrace{\left[\bar{\rho}\left(\widetilde{u_{i} h_{s}}-\tilde{u}_{i} \tilde{h}_{s}\right)\right.}_{\begin{array}{c}
\text { subgrid } \\
\text { enthalpy flux }
\end{array}}]
\end{gathered}
$$




$$
\frac{\partial \bar{\rho} \tilde{Y}_{k}}{\partial t}+\frac{\partial \bar{\rho} \tilde{u}_{i} \tilde{Y}_{k}}{\partial x_{i}}-\frac{\partial}{\partial x}\left(\bar{\rho} D \frac{\partial \tilde{Y}_{k}}{\partial x_{i}}\right)=-\frac{\partial}{\partial x_{j}}[\underbrace{\left[\bar{\rho}\left(\widetilde{u_{i} Y_{k}}-\tilde{u}_{i} \tilde{Y}_{k}\right)\right.}_{\begin{array}{c}
\text { subgrid } \\
\text { species flux }
\end{array}}]
$$

where $h_{s}, \lambda, D$, and $Y_{k}$ are the sensible enthalpy, thermal conductivity, mass diffusivity, and mass fraction of species $k$, respectively. As combustion is not being modelled in these simulations, there is no energy source term in equation (3) and equation (4) is strictly a transport equation for a non-reactive scalar used for visualization. The subgrid stress tensor is denoted as $\tau_{i j}$. The overline and tilde symbols refer to filtered and favre-averaged quantities, respectively. A favre-averaged variable is defined as:

$$
\tilde{\vartheta}=\frac{\overline{\rho \vartheta}}{\bar{\rho}}
$$

The resolved viscous stress tensor, $\tilde{\sigma}_{i j}$, and the resolved strain rate, $\tilde{S}_{i j}$, are defined as:

$$
\begin{gathered}
\tilde{\sigma}_{i j}=\mu\left(2 \tilde{S}_{i j}-\frac{2}{3} \frac{\partial \tilde{u}_{k}}{\partial x_{k}} \delta_{i j}\right) \\
\tilde{S}_{i j}=\frac{1}{2}\left(\frac{\partial \tilde{u}_{i}}{\partial x_{j}}+\frac{\partial \tilde{u}_{j}}{\partial x_{i}}\right)
\end{gathered}
$$

These governing equations are complemented by the filtered equation of state:

$$
\bar{p}=\bar{\rho} R \tilde{T}
$$

Although the gas constant, $R$, is composition dependent, the simulation results presented in this study correspond to a uniform mixture of stoichiometric methane-air. As is described later in this section, the purpose of using multiple species of equal composition is to aid in the visualization of the flow structures. The subgrid terms on the right-hand-side of equations (2)-(4) are modeled using a subgrid viscosity, $\mu_{\mathrm{SGS}}$, and a gradient diffusion approximation $[11,12]$ :

$$
\begin{gathered}
\underbrace{\bar{\rho}\left(\widetilde{u_{i} u_{j}}-\tilde{u}_{i} \tilde{u}_{j}\right)}_{\tau_{i j}}=-2 \mu_{\mathrm{SGS}} \tilde{S}_{i j} \\
\bar{\rho}\left(\widetilde{u_{i} h_{s}}-\tilde{u}_{i} \tilde{h}_{s}\right)=-\frac{\mu_{\mathrm{SGS}} C_{p}}{P r_{\mathrm{SGS}}} \frac{\partial \tilde{T}}{\partial x_{j}} \\
\bar{\rho}\left(\widetilde{u_{i} Y_{k}}-\tilde{u}_{i} \tilde{Y}_{k}\right)=-\frac{\mu_{\mathrm{SGS}}}{S c_{\mathrm{SGS}}} \frac{\partial \tilde{Y}_{k}}{\partial x_{j}}
\end{gathered}
$$

where $P r_{\mathrm{SGS}}=0.85$ and $S c_{\mathrm{SGS}}=0.7$ are the subgrid Prandtl number and subgrid Schmidt number, respectively.

The two subgrid models that were used to approximate the subgrid viscosity were the standard SmagorinskyLilly model and the dynamic Smagorinsky-Lilly model. To remove the effects of the subgrid model, simulations were also undertaken without any subgrid model. In the standard Smagorinsky-Lilly model, the subgrid viscosity is calculated from [13]:

$$
\begin{gathered}
\mu_{\mathrm{SGS}}=\bar{\rho} L_{s}{ }^{2}|S| \\
L_{s}=\min \left(\kappa y_{n}, C_{s} \Delta\right) \\
|S|=\sqrt{2 \tilde{S}_{i j} \tilde{S}_{i j}}
\end{gathered}
$$

where $L_{s}, \kappa, y_{n}$ and $C_{s}$ are the subgrid turbulence length scale, von Kármán constant, normal distance to the wall, and the Smagorinsky constant, respectively. Although the Smagorinsky constant has been calibrated 
to $C_{s}=0.17$ for the decay of homogeneous isotropic turbulence in the inertial subrange, the main weakness of this model is the sensitivity of $C_{s}$ to the grid resolution and type of flow regime [14]. In simulations using the standard Smagorinsky-Lilly model, the default value for the model constant was used $\left(C_{s}=0.1\right)$ [15]. Ideally, the constant, $C_{s}$, should vary in space and time as a function of $\Delta / l_{T}$, where $l_{T}$ is the integral length scale of turbulence [16]. The dynamic Smagorinsky-Lilly model allows the value of $C_{s}$ to vary spatially and temporally to better model the effects of turbulence on local flow conditions $[17,18]$. If $C_{s}$ is constrained to a single positive value, as done in the standard Smagorinsky-Lilly subgrid model, the energy transfer direction will be limited from large scales to small scales of turbulence. Although the energy transfer or subgrid scale dissipation is positive on average, intermittently, there are local regions of reversed energy transfer (backscatter) from the small scales back to the large scales. The dynamic model relies on the comparison of subgrid stresses calculated at two distinct filter widths at the same temporal and spatial position within the computational domain. The difference between the subtest-scale stress, $T_{i j}$, and the test-scale average of the subgrid stress, $\hat{\tau}_{i j}$, is defined as $L_{i j}$ :

$$
L_{i j}=T_{i j}-\hat{\tau}_{i j}=\widehat{\tilde{u}_{i} \tilde{u}_{j}}-\hat{\tilde{u}}_{i} \hat{\tilde{u}}_{j}
$$

$L_{i j}$ represents the resolved components of the stress tensor associated with scales of motion between the test scale and the grid scale and can be approximated as [17]:

$$
L_{i j}^{*}=2 C_{s}{ }^{2}\left(\hat{\Delta}^{2}\left|\hat{\tilde{S}}^{\mid} \hat{\tilde{S}}_{i j}-\Delta^{2}\right| \widehat{\widetilde{S} \mid \tilde{S}_{i j}}\right)
$$

Values of $C_{s}$ can be calculated to minimize the error of $L_{i j}-L_{i j}^{*}$, and therefore will correspond to more accurate levels and directions of energy transfer at the subgrid level. A least squares method of minimizing this error is defined as:

$$
C_{s}^{2}=\frac{\left\langle L_{i j} L_{i j}^{*}\right\rangle}{\left\langle L_{i j}^{*} L_{i j}^{*}\right\rangle}
$$

where \langle\rangle denotes spatial averaging in homogenous directions. Although this model appears to be more robust than the standard Smagorinsky-Lilly model by allowing $C_{s}$ to vary spatially and temporally, the model has difficulty in averaging in homogenous directions when implemented in complex geometries [19].

Equations (1)-(4) were solved using the segregated pressure-based solver of the commercial computational fluid dynamics software, ANSYSß Fluent v.6.3. Each simulation was run in parallel on twelve UltraSPARC-IV, $1.5 \mathrm{GHz}$ processors at the Canadian High Performance Computing Virtual Laboratory (HPCVL). During each timestep each equation was solved sequentially and then subsequently updated using a pressure-correction equation [15]. The pressure-correction equation was implemented through the Semi-Implicit Method for Pressure-Linked Equations (SIMPLE) modified for unsteady simulations [20]. The SIMPLE pressure-velocity coupling scheme was used with under-relaxation factor values of $0.3,1,1,0.7$, and 1 for the pressure, density, body forces, momentum, and energy terms, respectively. Under-relaxation is used to slow the change of a variable as the governing equations are iteratively solved in order to provide stability to the process and ensure fast convergence rates. The pressure interpolation scheme and convective terms were discretized using a bounded central difference scheme, while diffusion terms were calculated using a central differencing scheme. The bounded central differencing scheme is a pure central differencing scheme that is blended with a first order upwind scheme in the event of numerical instability, which is monitored through a convection boundedness criterion [21]. Second order implicit time integration was used to discretize time derivatives.

The computational domain, shown in figure 1, corresponds to a volume spanning over two obstacle pairs ahead of the flame front. Note that the flame shown in the schematic was not modeled and was only provided as a reference to the experiments. The upstream side of the domain was positioned approximately $33.5 \mathrm{~cm}$ downstream of the ignition source. The obstacle spacing was equal to the channel height of $H=7.62 \mathrm{~cm}$ and the length of the computational domain was varied from $L / H=1.4$ to $L / H=2.4$. The domain width was varied from a pure $2 \mathrm{D}$ simulation to a maximum width of $W / H=0.67$. The channel width in the experiment was $W / H=1$. Note that the helium injection into the channel was not simulated. Instead, the initial conditions included a pocket of helium gas positioned between two consecutive obstacles pairs. A passive scalar in the gas was initialized to $C=0$ and $C=1$, upstream and downstream of the second 


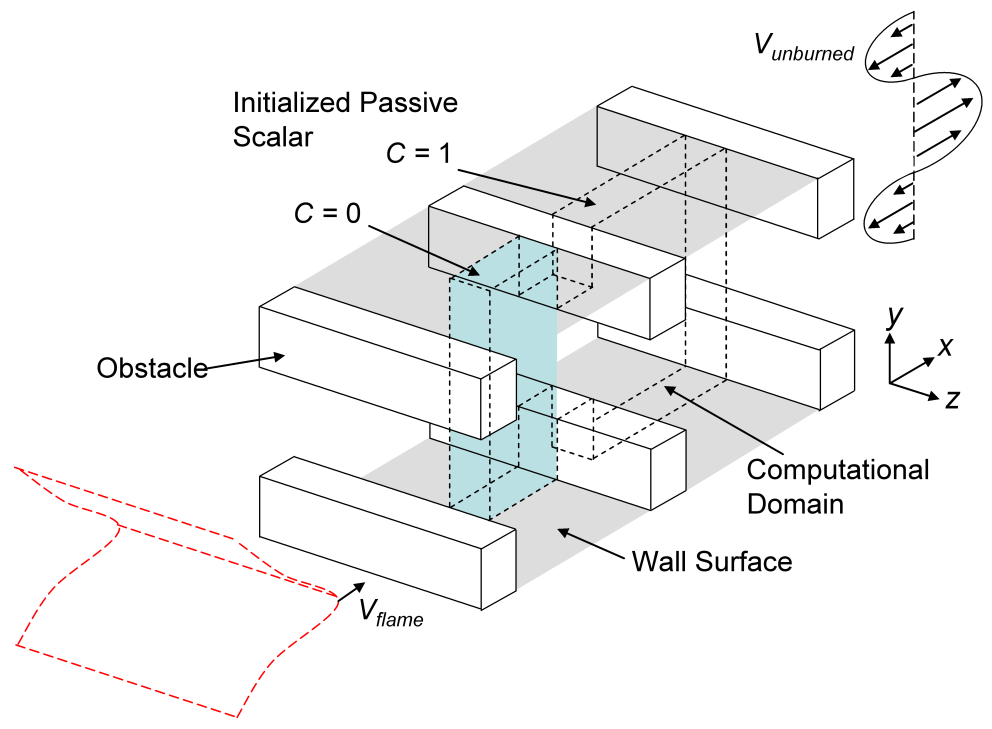

Figure 1: Computational domain $(\mathrm{BR}=0.5)$.

obstacle, respectively. $C=0$ corresponds to a pure methane-air mixture and $C=1$ corresponds to helium tracer gas as shown in figure 1. In these simulations, however, all gas properties, including the density and viscosity, were not affected by values of $C$ and correspond to the methane-air mixture. The convection of the interface between $C=0$ and $C=1$ was simulated merely to visualize development of the unburned gas and to allow comparisons with the methane-air/helium interface that was observed in the experiment. Results from simulations modeling the effects of helium concentration levels on the methane/helium gas interface indicate that the density gradient which occurs in the experiment does not affect the development of the flow field.

A mass flow inlet boundary condition was imposed on the shaded region in figure 3 , which corresponds to a streamwise position of $0.5 \mathrm{H}$ upstream of the obstacle's surface. The specification of the mass flux was obtained experimentally by tracking the convection of the methane-air/helium gas interface across the top of the obstacle's surface (figure 2).

An exponential equation was curve fitted to this data and extrapolated to obtain the mass flux at later times. For stoichiometric methane-air at an initial pressure of $p_{i}=47 \mathrm{kPa}$ and initial density of $\rho=0.535$ $\mathrm{kg} / \mathrm{m}^{3}$, the unsteady mass flux, $\dot{m}$, is specified as:

$$
\dot{m}=A \rho t^{n}
$$

where $t$ is the simulation time in seconds. $A$ and $n$ are constants obtained from the experiment with values of 12,400 and 1.7, respectively. Equation (18) does not account for any spatial variations in the mass flux including any turbulence that may have been generated upstream of the inflow boundary. Since combustion was not modelled, the duration of the simulation spanned from ignition to a time corresponding to the arrival of the flame at the entrance of the computational domain. Implementation of equation (18) into Fluent v.6.3 was facilitated through a User Defined Function (UDF).

The top and bottom boundaries shown in figure 3 are zero-slip wall surfaces that specify zero mass flux and zero velocity. The grid density near wall surfaces was sufficiently fine to resolve the laminar sublayer $\left(y^{+}<1\right)$. The wall shear stress, $\tau_{w}$, was obtained from the linear relationship:

$$
\tau_{w}=\mu \frac{\tilde{u}}{y}
$$

where $\tilde{u}$ is the resolved near-wall velocity. All of the subgrid terms in equations (2)-(4) are considered to be negligible in the laminar sublayer and were suppressed near the wall surfaces through equation (13). 


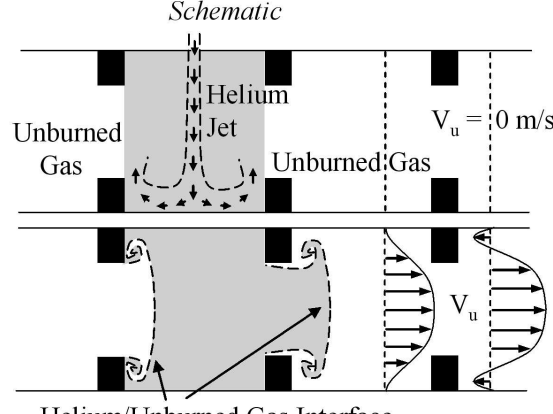

Helium/Unburned Gas Interface
Schlieren Images

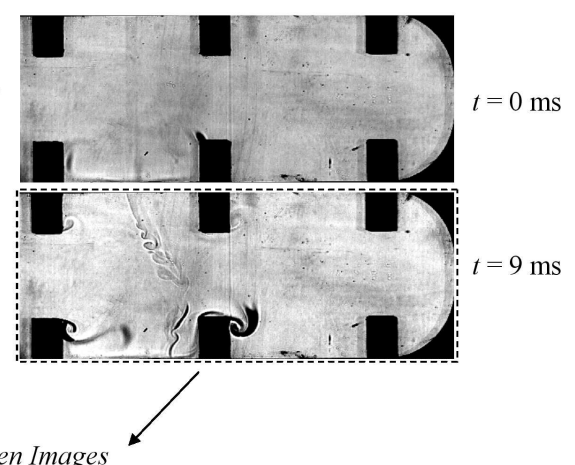

Enlarged Schlieren Images

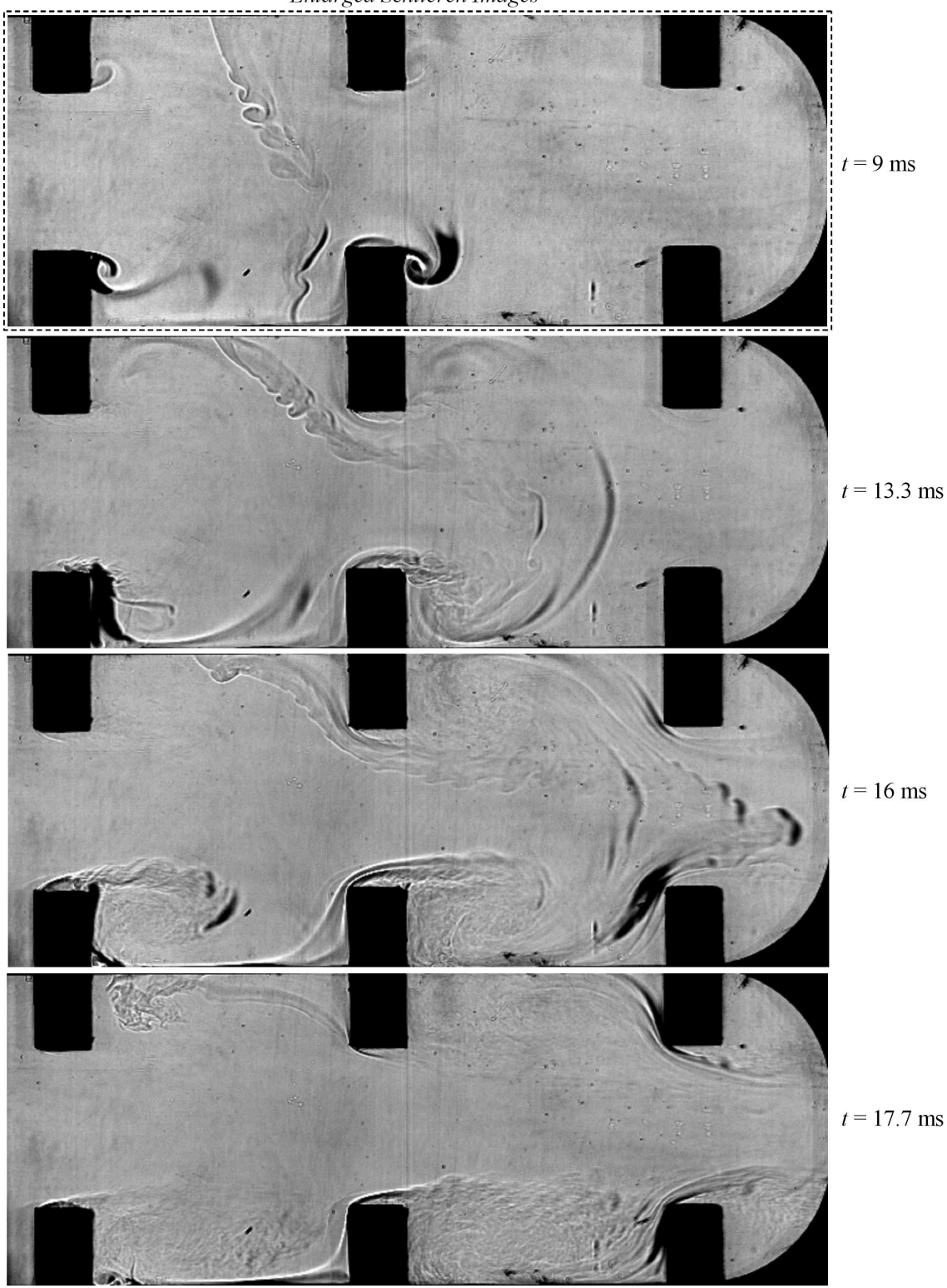

Figure 2: Schlieren photographs and schematic of the development of the unburned gas flow field ahead of the flame using helium gas as a tracer. Adapted from [10]. 


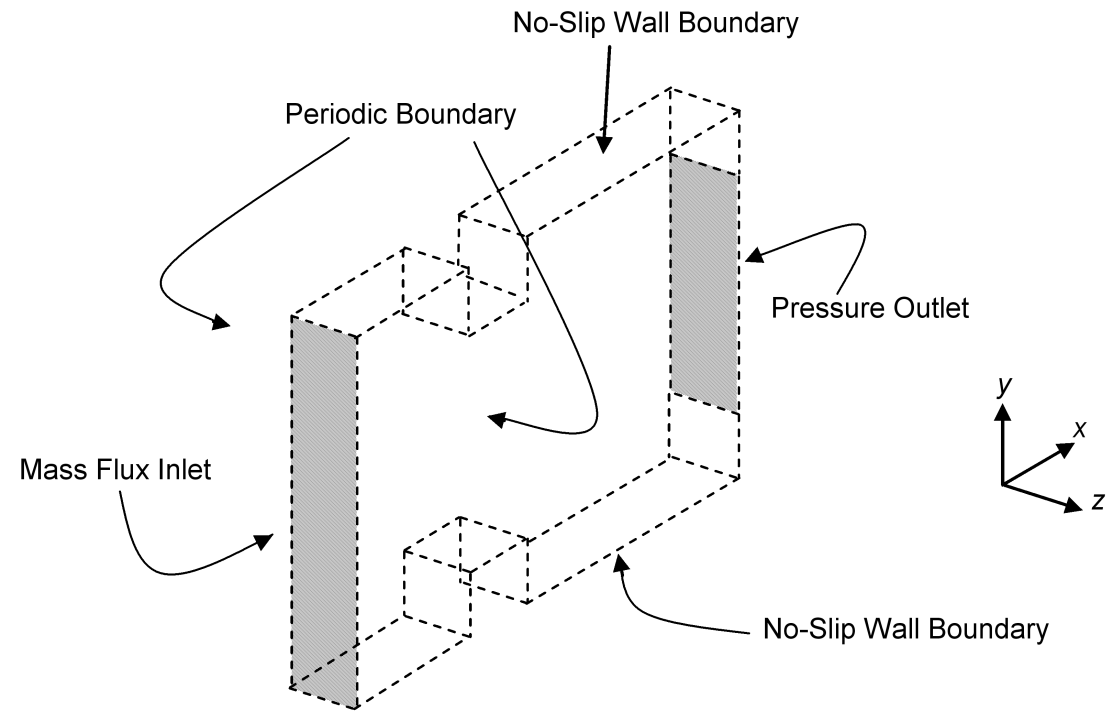

Figure 3: Boundary conditions for $\mathrm{BR}=0.5$ case $(W / H=0.167)$.

At the outflow boundary, the static pressure was set to the initial pressure, $p_{i}$. The two side surfaces were designated as periodic boundaries. The base grid has a node spacing of $\Delta=0.6 \mathrm{~mm}$, which corresponds to $H / \Delta=127$ and a total of one million nodes in the entire domain $(W / H=0.67, L / H=1.4)$. In these simulations, the filter size is equivalent to the node spacing. Hexahedral cells with uniform node spacing in each direction were used in the domain interior and high aspect ratio cells were used near the wall surfaces. To allow for a smooth transition between the wall surface and pressure outlet at the downstream face of the computational domain, the high aspect ratio cells were extended over the pressure outlet and inflow boundary.

\section{Initial Laminar Vortex Development}

Based on the experiments [10], it appears that the initial laminar rollup of the vortex was two-dimensional. Therefore, a highly resolved two-dimensional simulation $(W / H=0)$ was undertaken to gain insight into its development. Figure 4 shows the distribution of $C$ and vorticity on a two-dimensional grid, which was dynamically refined. The adaptation scheme dynamically refined and coarsened the grid based upon the local magnitude of the gradient of $C$, wall $y^{+}$, and the resolved strain-rate. The base grid for the dynamic adaption case corresponds to a spacing of $H / \Delta=127$. Grid volumes marked for refinement at each time step were uniformly split into smaller volumes for a maximum of three refinement levels, which corresponds to a minimum grid spacing of $\Delta=75 \mu \mathrm{m}$ or $H / \Delta=1016$. Shown on the left side of figure 4a is a plot of the $C$ contour at a time of $t=8.7 \mathrm{~ms}$ where the position of the $C=0.5$ contour is visible. The corresponding computational grid resulting from dynamic refinement is shown on the right side of figure 4a. This algorithm operated at each time step and the grid was repartitioned every 200 time steps $(1.3 \mathrm{~ms})$ to properly loadbalance each of the twelve processors used in this simulation.

Contours of $C$ in figure $4 \mathrm{~b}$ show how the shape of the initially planar $C=0.5$ iso-surface became distorted in the $x-y$ plane. Note the $C$ contours are overlaid on the instantaneous stream-traces. A large deformation in the $C=0.5$ iso-surface was visible near the obstacle's top surface $(t=6.7 \mathrm{~ms}$ ) due to the transverse $(y)$ distribution of streamwise velocity in the boundary layer. Due to the velocity deficit in the boundary layer, the core flow near the obstacle accelerated above the centerline velocity. This resulted in an indentation in the $C=0.5$ iso-surface at the channel centerline (left image in figure 4a). Separation of the

flow occurred initially at the obstacle's leading and trailing edges, which are shown as clockwise rotating fluid (red contours of vorticity, figure 4c). As the simulation progressed, the strength and size of these separated 
C $\quad \omega_{z}\left(\mathrm{~s}^{-1}\right)$

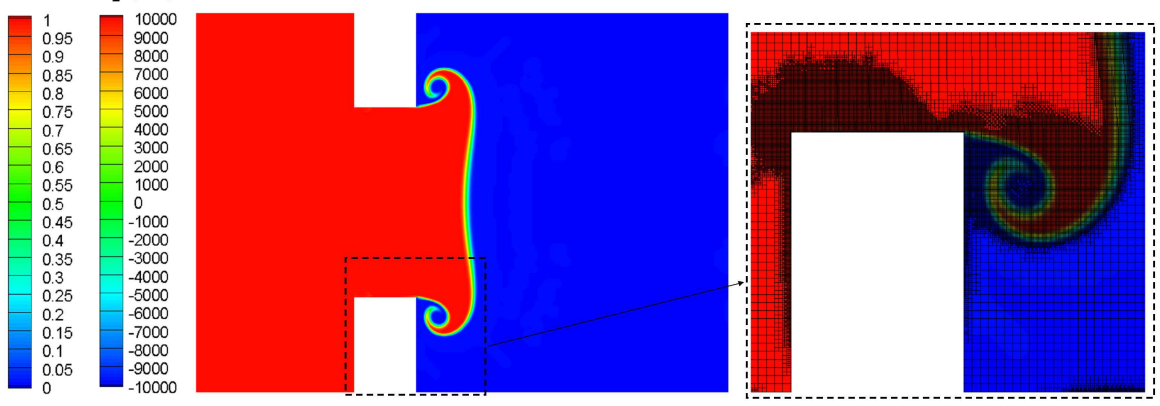

a)
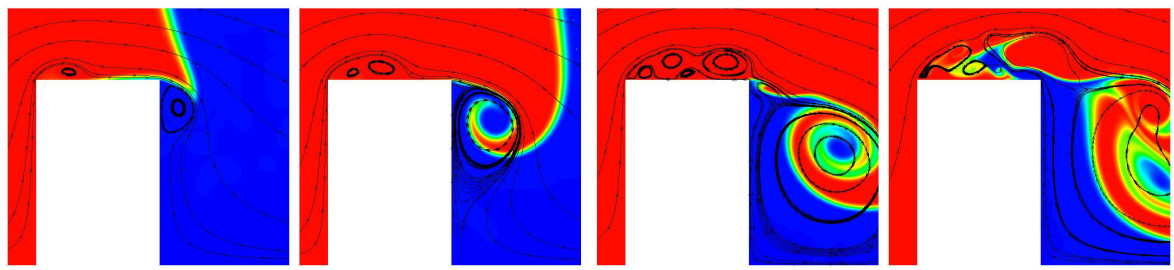

c)
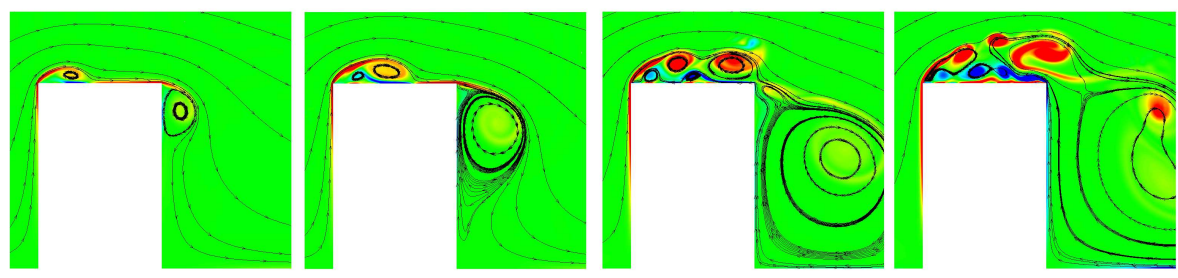

$t=6.7 \mathrm{~ms}$

$t=8.7 \mathrm{~ms}$

$t=11.3 \mathrm{~ms}$

$t=13.3 \mathrm{~ms}$

Figure 4: Laminar rollup of vortex and instabilities in shear layer shown on a dynamically adapted grid. a) $C$ distribution shown on the full field of view with the computational grid at $t=8.7 \mathrm{~ms}$. (contour legends shown on left, $\mathrm{BR}=0.5, x-y$ plane). b) Evolution of $C$ distributions with over-laid stream-traces. c) Evolution of the distribution of vorticity (clockwise $=$ red, counterclockwise $=$ blue) with overlaid stream-traces. 
zones increased, which caused smaller counter-rotating vortices (blue contours) to form near the obstacle's tips $(t=8.7 \mathrm{~ms})$. At $t=11.3 \mathrm{~ms}$, the complex dynamic of rotating and counter-rotating vortices were visible along the obstacle's top surface and the core flow was no longer able to reattach near the obstacle's trailing edge. Furthermore, at $t=13.3 \mathrm{~ms}$, large vortices began to shed away from the obstacle and were convected along the shear layer created by the initial vortex rollup. Reversed flow along the obstacle's top surface ( $t=13.3 \mathrm{~ms}$, figure $4 \mathrm{~b}$ ) allowed fluid marked with $C=0$ (blue contour) to convect back towards the obstacle's leading edge. At this point, small scale structures associated with the vortices became visible in the distribution of $C$. Stream-traces in the core flow were visibly distorted as they passed through the perimeter of these vortices. The shape of these stream-traces is important as they play a large role in determining the shape of the flame front as it propagates within the core. As the intensity of these vortices increases, span-wise instabilities within the flow will cause $x$ - and $y$ - components of vorticity to form, which are not possible to capture in a two-dimensional simulation. The next section presents simulation results based on a three-dimensional computational domain.

\section{Three-Dimensional Flow Field Development}

Figure 5 shows the three-dimensional flow development corresponding to a simulation using 0.5 BR obstacles. In this simulation, the standard Smagorinsky-Lilly model was used. The simulation has a corresponding width of $W / H=0.67$, length of $L / H=1.4$, and a uniform grid spacing of $H / \Delta=127$. Visualization of the interface was accomplished by tracking a $C=0.5$ iso-surface over time, which shows the two-dimensional formation of the recirculation zone downstream of an obstacle. Stream-traces distributed near the bottom channel surface indicate that for early simulation times $(t=13.3 \mathrm{~ms})$ the flow field contracted and expanded upstream and downstream of the obstacles. This oscillating streamwise velocity was responsible for the oscillating flame-tip velocity observed in the experiment [10]. However, at later times $(t=20 \mathrm{~ms})$ the shear layer that developed spanned the distance between the obstacles. Consequently, the core flow did not expand past the obstacles as is indicated by the roughly horizontal stream-traces. Levels of small scale turbulence are indicated by the presence of subgrid stresses, which are a product of subgrid viscosity and resolved strain-rate as indicated in equation 9 . The subgrid viscosity ratio, $\mu_{\mathrm{SGS}} / \mu$, is shown as contours on the $C$ $=0.5$ iso-surface as it is equivalent to the ratio of the local subgrid stress to the local viscous stress. Low levels (blue color) of subgrid viscosity ratio were observed along the shear layer and within the core flow between the obstacles. As expected, the largest levels (red color) of subgrid viscosity ratio were observed near the leading edge of each obstacle where the level of the resolved strain-rate was at a maximum. After the recirculation zone spanned the distance between the obstacles $(t=20 \mathrm{~ms})$, the level of subgrid viscosity exceeded five times the magnitude of the molecular viscosity and span-wise ( $z$-direction) variations began to occur in the $C=0.5$ iso-surface. Small deformations along the $C=0.5$ iso-surface were due to the interaction with the discrete vortices that were convected along the shear layer. This is observed as small clumps of $z$-vorticity as shown on the $x-y$ plane on the left periodic boundary.

An iso-surface of $x$-vorticity, shown in figure 6 , displays the formation of small scale vortical structures in the streamwise direction that are concentrated in the shear layer. Vorticity components in each of the three spatial dimensions are an indication of a transition to turbulent flow. The characteristic size and vorticity magnitude of the streamwise vortices predicted in the simulations were very small compared to values observed from $z$-vorticity field. As a result, all major distortions of the flow still occurred in the $x-y$ plane, which explains the absence of major span-wise variation in the streamlines near the end of the simulation $(t=20 \mathrm{~ms})$.

\section{Effect of Obstacle Blockage Ratio on the Unburned Gas Flow}

One of the main objectives of this study is to understand the effect of the obstacle blockage ratio on the unburned gas flow field. Qualitatively, figure 7 shows the convection of the passive scalar, $C$, for three different blockage ratios and is compared to the methane-air/helium gas interface observed in the experimental schlieren images. In these simulations, $C_{s}$ was calculated using the dynamic Smagorinsky model and the grid resolution corresponded to $H / \Delta=127$. Based on the results of the previous section, which showed that cross-stream effects were mild, a longer domain $(L / H=2.4)$ was used in these simulations at the expense 

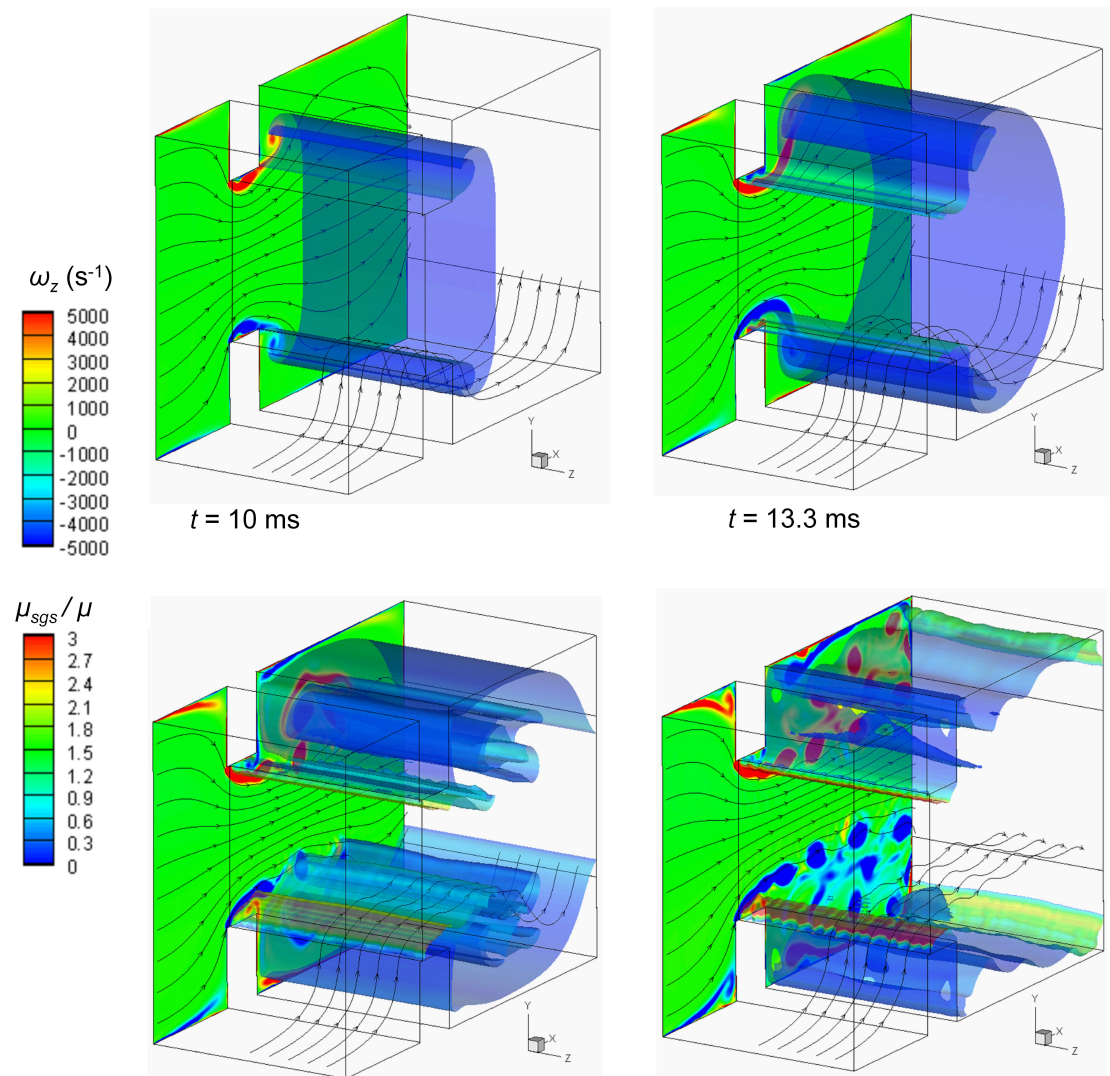

$t=16.7 \mathrm{~ms}$

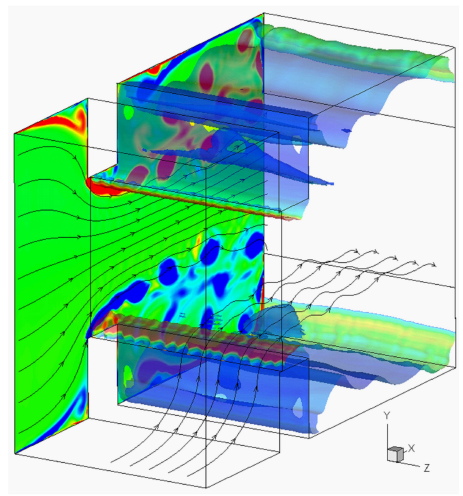

$t=20 \mathrm{~ms}$

Figure 5: 3D flow field development $(\mathrm{BR}=0.5$, standard Smagorinsky). Distribution of subgrid viscosity ratio is shown as contours on $C=0.5$ iso-surface. Distribution of $z$ vorticity is shown on $x-y$ plane. Stream-traces are distributed along $y$ axis at the $-z$ extent of domain and near bottom wall surface.
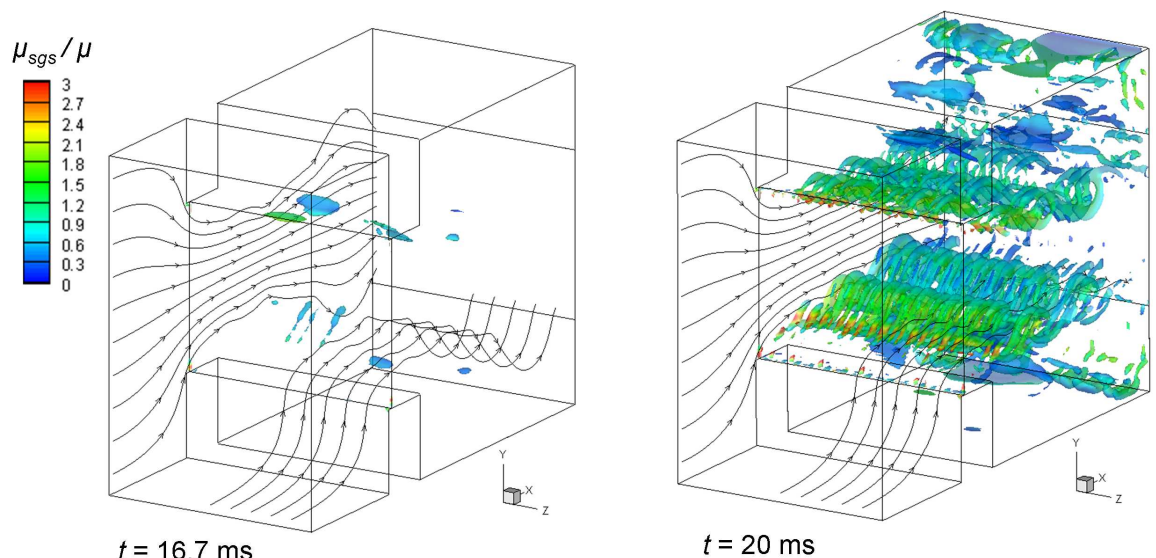

Figure 6: Iso-surface of $x$-vorticity $\left(\omega_{x}=1000 s^{-1}, \mathrm{BR}=0.5\right.$, standard Smagorinsky). Contours of subgrid viscosity is shown on $x$-vorticity iso-surface. Stream-traces are distributed along $y$ axis at the $-z$ extent of domain and near bottom wall surface. 
of the domain width $(W / H=0.167)$. The unsteady mass flux at the inflow boundary was identical for each simulation and is defined in equation (18). The early flow development was predicted extremely well as the laminar vortex grew behind each obstacle. One observation was that the vortex centerline position advanced quicker in simulations with larger BR obstacles due to the higher core flow velocity through the opening between obstacles. Also, for the smaller BR cases, the recirculation zones reached the channel surface earlier, thus affecting the roll-up. Due to the higher core velocity corresponding to the higher BR cases, the recirculation zones became turbulent earlier, which is a phenomena predicted in the simulations and confirmed in the experiment.

Figure 8 shows the evolution of centerline streamwise velocity for six different simulation times on the same computational domain $(L / H=2.4, H / \Delta=127)$. A smooth rise in streamwise velocity before each obstacle was observed in each simulation, with the largest rise occurring in the largest BR case. Following the rise, a drop in velocity after each obstacle occurred, which resulted in a sinusoidal type oscillation in the centerline velocity. The duration of the oscillation was longer in the lower obstacle BR simulations since the recirculation zones grew at a slower rate compared to the higher obstacle BR simulations. For example, at $t=16.7 \mathrm{~ms}$, the recirculation zone corresponding to the $\mathrm{BR}=0.67$ case grew to span the distance between the obstacles, while the recirculation zone corresponding to the $\mathrm{BR}=0.33$ case only grew to approximately half the distance between the obstacles (figure 7).

Instabilities produced near the obstacle's leading edge caused a laminar-to-turbulence transition to occur. This transition first occurred in the larger obstacle BR simulations $(t=13.3 \mathrm{~ms})$ due to the high streamwise velocity near the obstacle's leading edge and the large adverse pressure gradient downstream of the obstacle's trailing edge. Small perturbations in the centerline velocity shown in figure $8(t=15.3 \mathrm{~ms}, \mathrm{BR}=0.67)$ are due to the small vortices that formed at the obstacle's leading edge and which convected along the shear layer. As the size of these vortices increased, the amplitude of centerline velocity oscillations also increased.

The proximity of vortex shedding to the centerline of the channel was much smaller for the large BR case, and therefore the effect that the vortices had on the centerline velocity was amplified. Instabilities in the centerline velocity predicted by the $\mathrm{BR}=0.5$ simulation did not start until approximately $t=16.7 \mathrm{~ms}$. The larger opening between the obstacles allowed for a lower centerline velocity in the $\mathrm{BR}=0.5$ case, which delayed the transition to a turbulent vortex. In the obstacle $\mathrm{BR}=0.33$ case, the oscillations in centerline velocity appeared to be unaffected for most of the simulation. For the $\mathrm{BR}=0.5$ and $\mathrm{BR}=0.67$ cases, the lengthening of the shear layer at later simulation times inhibited flow expansion, which allowed the centerline velocity to remain high downstream of obstacles $(t=22.7 \mathrm{~ms})$.

Oscillations in the flame velocity followed a sinusoidal profile at early times as predicted by the simulations (figure 9). Since the centerline velocity of the unburned gas is much larger than the laminar burning velocity of methane-air, convection of the flame-tip at the centerline is dictated by the velocity in the unburned gas. At later times, however, oscillations in flame velocity still occurred in regular intervals between the obstacles prior to the formation of shock waves [22]. It is possible that changes in the volumetric burning rate did not follow the smooth rise in mass flux that was specified at the inflow at these later simulations times. Flame interaction with the turbulent flow field is expected to cause changes in the volumetric burning rate. Therefore, future work involving the simulation of flame propagation at these speeds is required to understand the nature of these oscillations.

\section{Sensitivity Analysis}

The effects of grid resolution, domain shape, and subgrid model on the solution results were investigated. A steady and unsteady flow analysis showed that the solution is insensitive to grid resolution levels at both early and later stages of flow development. Figure 10 shows a qualitative comparison of the evolution of $C$, where $C=0$ is blue and $C=1$ is red, for simulations with three different grid resolutions $(H / \Delta=$ $127, H / \Delta=171, H / \Delta=254)$. The domain width in these simulations corresponds to $W / H=0.03 . U_{\mathrm{inf}}$ in these figures refers to the velocity at the inflow boundary. Initially, the overall structure of the vortex appears to be unaffected by the grid resolution level up to a simulation time of $t=13.3 \mathrm{~ms}$. The interface between $C=0$ and $C=1$ is sharper and the small instabilities responsible for vortex shedding near the obstacle leading edge are visible on the higher resolved grid $(H / \Delta=254)$. Even as instabilities within the shear layer developed and vortices began to shed from the obstacle's leading edge, the overall structure of 


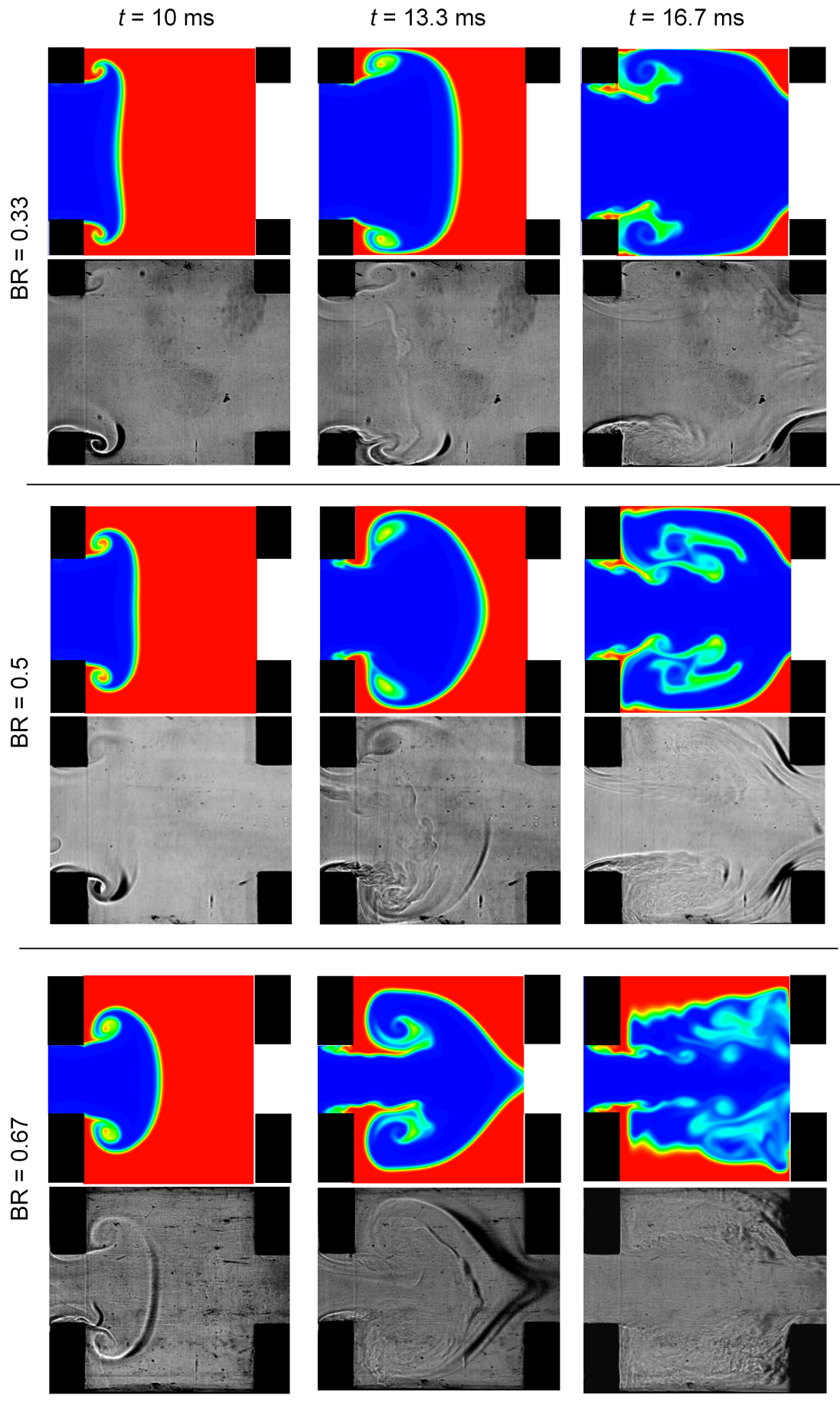

Figure 7: Effect of blockage ratio on the flow field development. Top images are contours of $C$ from simulations (dynamic Smagorinsky). Bottom images are evolution of helium/methane-air gas interface from experiment [10]. 

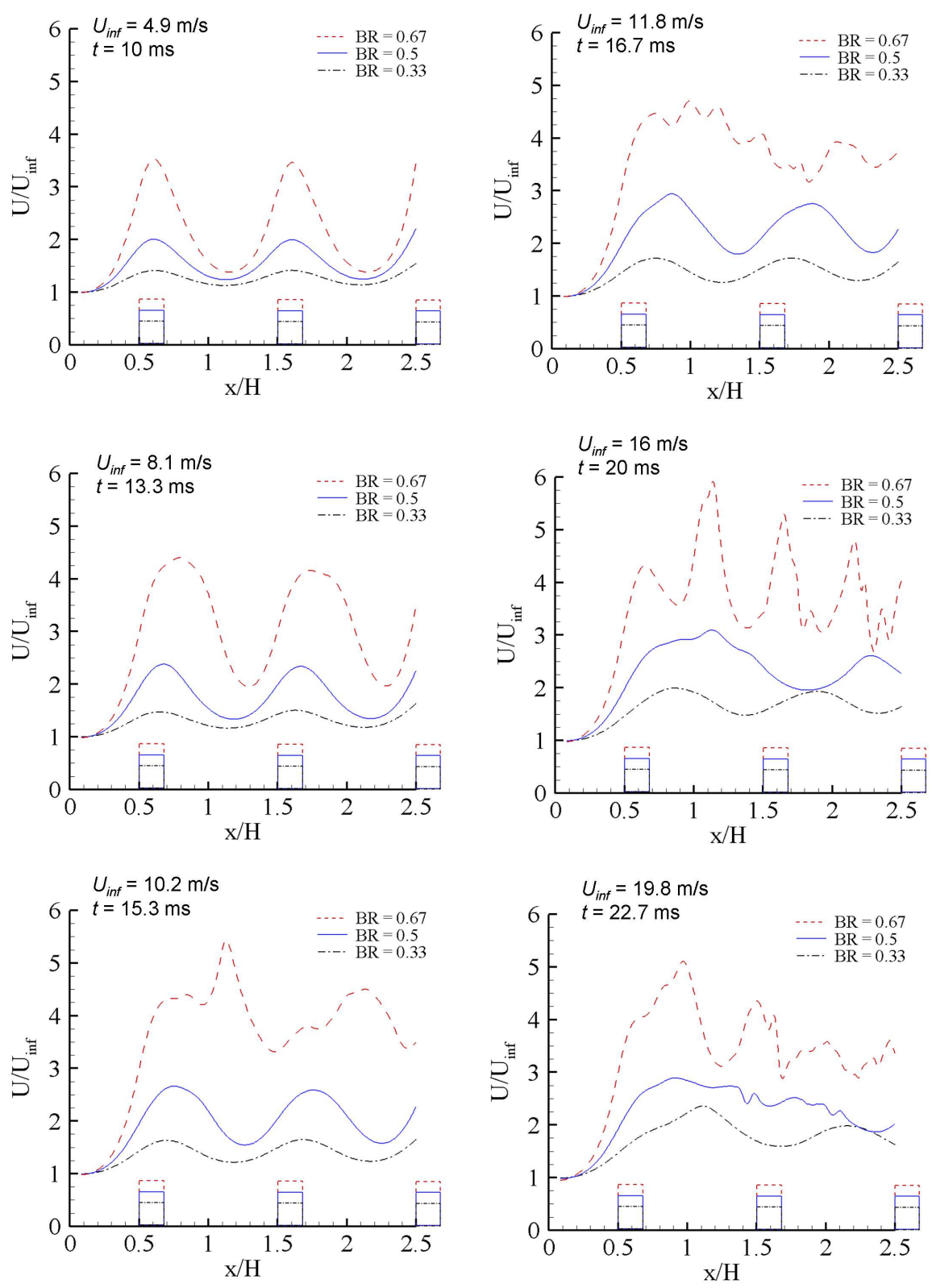

Figure 8: Effect of blockage ratio on the evolution of centerline velocity. 


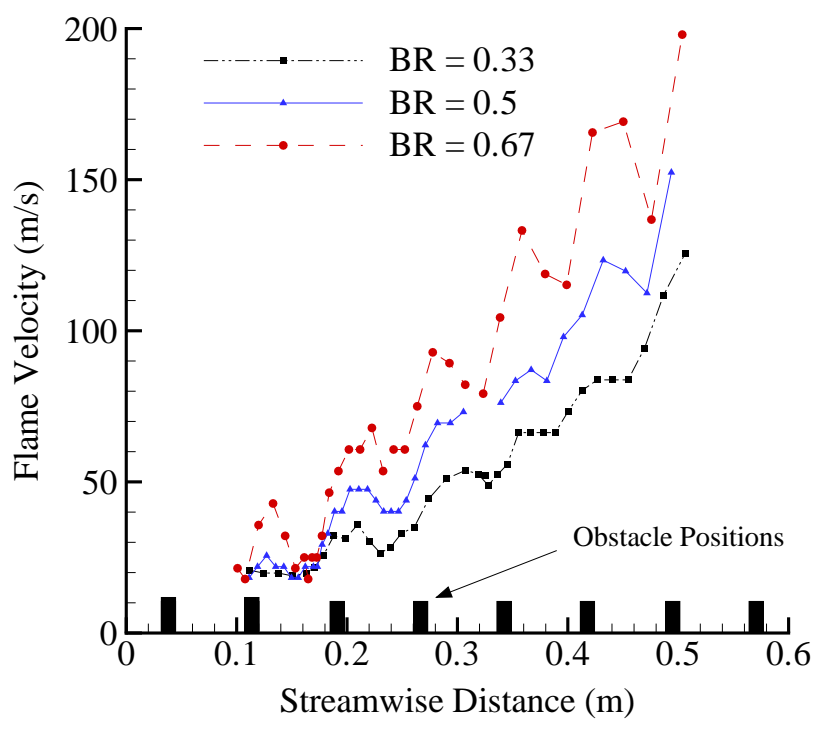

Figure 9: Oscillations in flame-tip velocity measurements for varying obstacle blockage ratio. Velocity measurements obtained from schlieren image sequence. Adapted from [10].

recirculation zone remained insensitive to the choice of grid resolution. Noticeable differences in the small scale structure, however, were visible as the grid resolution increased. Furthermore, when the shear layer extended between the obstacle's tips, the size and shedding frequency of the vortices was affected by the grid resolution. The shedding frequency increased by approximately 25 percent in simulations with the highest resolved grid $((H / \Delta=154))$ in comparison to simulations with the lowest resolved grid $(H / \Delta=127)$. The solution was found to be insensitive to the CFL number over a range of $0.125<\mathrm{CFL}<1$.

Variation of the domain width $(W / H=0.03, W / H=0.17, W / H=0.67)$ and the domain length $(L / H$ $=1.4, L / H=2.4$ ) had no impact on the solution at early simulation times as the initial laminar rollup was found to be two-dimensional and was unaffected by the position of the outflow boundary. As the transition to turbulence occurred within the shear layer, three-dimensional effects became important and the domain width began to have an effect on the distribution of $C$. Results from the smallest domain width case $(W / H$ $=0.03$ ) showed that the shedding frequency was approximately 25 percent lower than what was observed in simulations with larger domain widths. In addition, the size of each vortex that was convected along the shear layer increased in simulations using smaller domain widths. The structure of the recirculation zone downstream of the obstacle at $t=20 \mathrm{~ms}$ is very similar between the $W / H=0.17$ and $W / H=0.67$ cases, which indicates that $W / H=0.17$ is sufficient to accommodate three dimensional effects in the flow (figure 11). As the shear layer spanned the distance between the obstacles, the position of the outflow boundary began to affect the structure of the recirculation zone. As shown in figure 12, for example, the flow remained artificially symmetrical near the imposed pressure field at the outflow boundary $(t=20 \mathrm{~ms})$.

The subgrid model had no effect on the initial vortex rollup due to low levels of subgrid viscosity. As the shear layer spanned the distance between the obstacles, the dynamic subgrid model and no model $\left(C_{s}=0\right)$ predicted smaller vortices shed at a higher frequency than predicted by the standard Smagorinsky model. The Standard Smagorinsky-Lilly model often over-predicts levels of subgrid viscosity because the model assumes a constant flow of energy from the resolved scales of turbulence to the dissipative Kolmogorov scales at equilibrium conditions [17]. As the contribution of the subgrid model diminished (i.e. $C_{s}=0$ ), asymmetry was observed in the flow. However, stream-traces were not significantly affected by the asymmetry suggesting that the core flow was not largely affected by small scale turbulence (figure 13). 


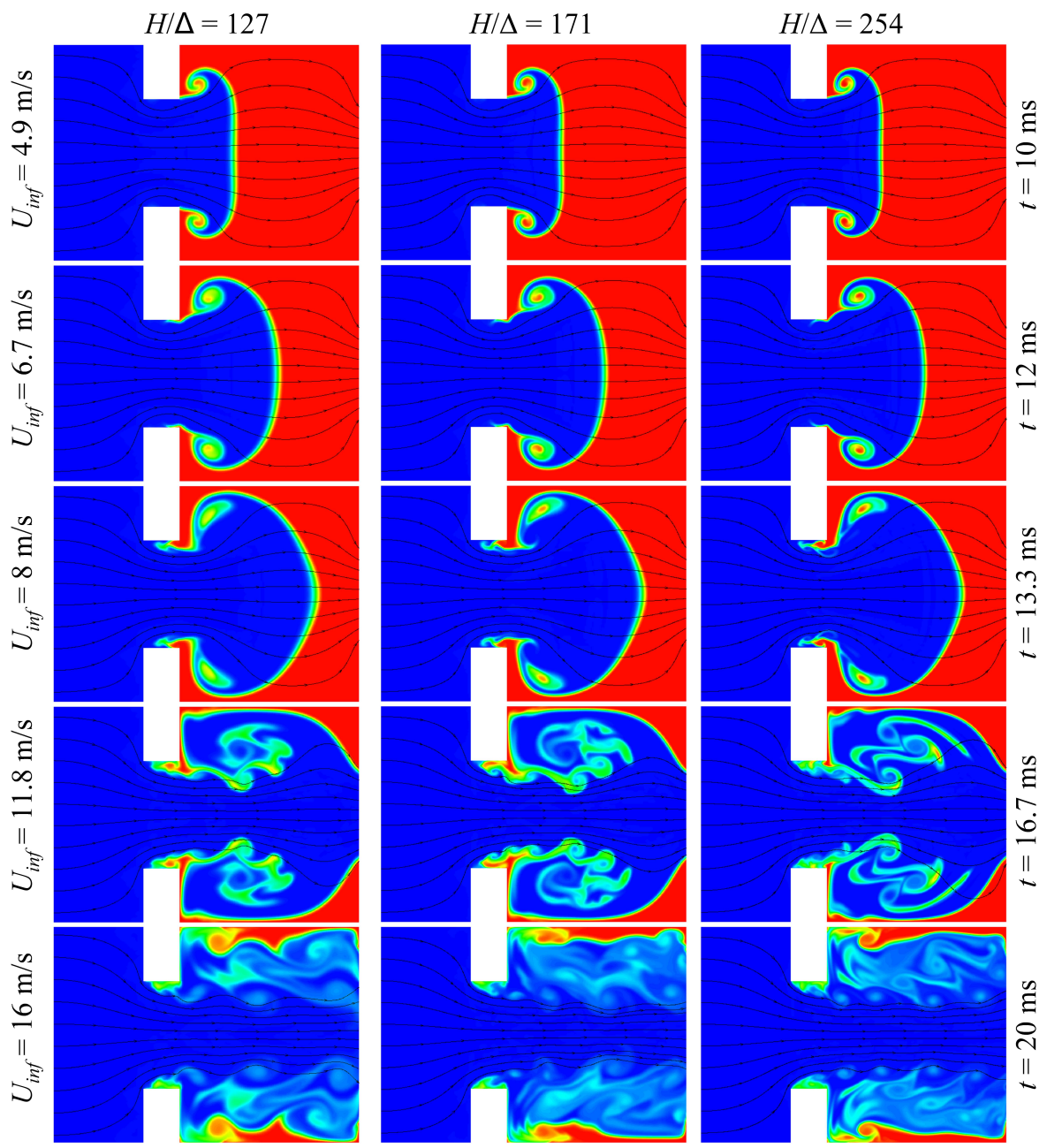

Figure 10: Effect of grid resolution on the distribution of $C$ ( $C=0$ shown in blue, $C=1$ shown in red). Contour legend shown in figure $4 . \mathrm{BR}=0.5$. Stream-traces overlaid onto contour plot.

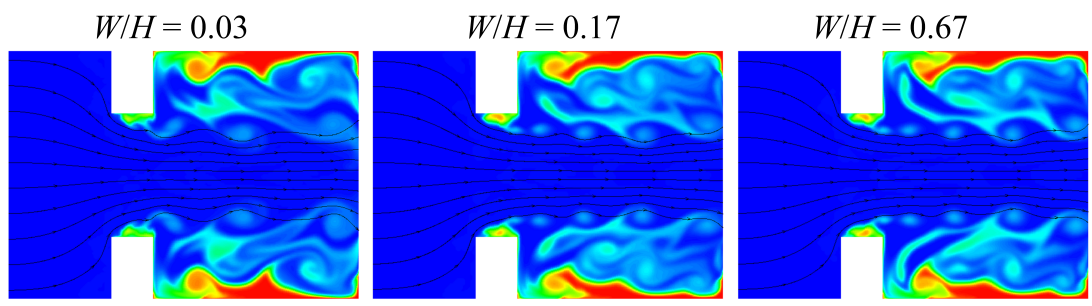

Figure 11: Effect of domain width on the distribution of $C$ ( $C=0$ shown in blue, $C=1$ shown in red). Contour legend shown in figure $4 . \mathrm{BR}=0.5$. Stream-traces overlaid onto contour plot $\left(t=20 \mathrm{~ms}, U_{\text {inf }}=\right.$ $16 \mathrm{~m} / \mathrm{s})$. 

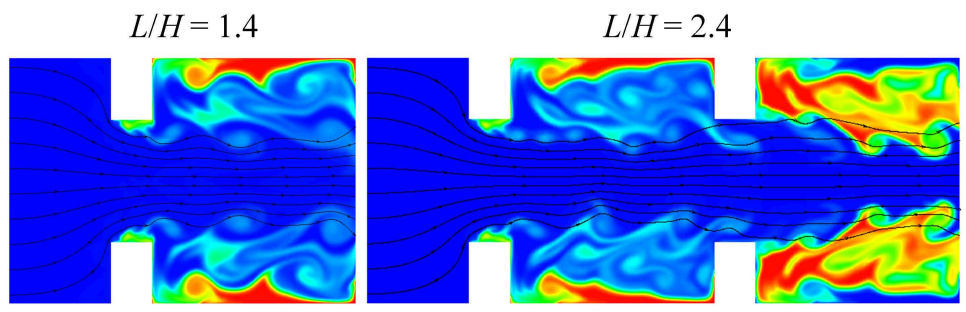

Figure 12: Effect of domain length on the distribution of $C$ ( $C=0$ shown in blue, $C=1$ shown in red). Contour legend shown in figure $4 . \mathrm{BR}=0.5$. Stream-traces overlaid onto contour plot $\left(t=20 \mathrm{~ms}, U_{\text {inf }}=\right.$ $16 \mathrm{~m} / \mathrm{s})$.
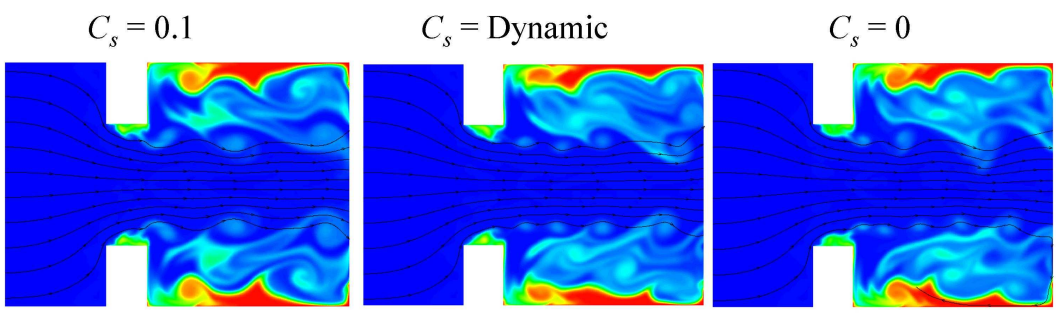

Figure 13: Effect of subgrid model on the distribution of $C$ ( $C=0$ shown in blue, $C=1$ shown in red). Contour legend shown in figure $4 . \mathrm{BR}=0.5$. Stream-traces overlaid onto contour plot $\left(t=20 \mathrm{~ms}, U_{\text {inf }}=\right.$ $16 \mathrm{~m} / \mathrm{s})$.

\section{Conclusions}

Numerical simulations of the unburned gas flow indicated that a transition from two-dimensional to threedimensional flow occurred roughly at the time corresponding to the lengthening of the recirculation zones to span the distance between obstacles. This point of transition coincided with instabilities produced near the obstacle's leading edge, which resulted in a transition to turbulence and subsequent shedding of vortices along the shear layer. Although not simulated, the development of these small flow structures results in the wrinkling of the flame surface, which increases the total flame area. High levels of small scale turbulence produced near the obstacle's leading edge were indicated by the large levels of subgrid viscous stresses. The flame front velocity oscillations that were observed in the experiments [10] could be explained by the flow contraction and expansion observed in the simulations. Results showed, however, that as the recirculation zones lengthened to span the distance between obstacles, the level of contraction and expansion of the core flow diminished even though the experiment showed continued centerline flame velocity oscillations. This suggests that the observed late-time flame front velocity oscillations were not caused solely by flow contraction and expansion, but by changes in the volumetric burning rate associated with the flame propagation that were not taken into account in the simulation.

\section{Acknowledgements}

This work was supported by the Natural Sciences and Engineering Research Council of Canada. Simulations were performed using the Canadian High Performance Computing Virtual Laboratory. 


\section{References}

[1] G. Ciccarelli and S. Dorofeev, Flame acceleration and transition to detonation in ducts, Prog. Energy Combust. Sci. 34 (2008), pp. 499-550.

[2] G.D. Roy, S.M. Frolov, A.A. Borisov, and D.W. Netzer, Pulsed detonation propulsion: challenges, current status, and future perspective, Prog. Energy Combust. Sci. 30 (2004), pp. 545-672.

[3] K.I. Shchelkin, Influence of tube roughness on the formation and detonation propagation in gas, J. Exp. Theor. Phys. 10 (1940), pp. 823-827.

[4] S.M. Frolov, Liquid-fueled, air-breathing pulse detonation engine demonstrator: operation principles and performance, J. Propuls. Power 22 (2006), pp. 1162-1169.

[5] P. Middha and O.R. Hansen, Predicting deflagration to detonation transition in hydrogen explosions, Process Saf. Prog. 27 (2008), pp. 192-204.

[6] V. Gamezo, O. Takanobu, and E.S. Oran Numerical simulations of flame propagation and DDT in obstructed channels filled with hydrogen-air mixture, Proc. Combust. Inst. 31 (2007), pp. 2463-2471.

[7] V. Bychkov, D. Valiev, and L. Eriksson, Physical mechanism of ultrafast flame acceleration, Phys. Rev. Lett. 101 (2008), pp. 1-4.

[8] V. Di Sarli, A. Di Benedetto, G. Russo, S. Jarvis, E.J. Long, and G.K. Hargrave, Large eddy simulation and PIV measurements of unsteady premixed flames accelerated by obstacles, Flow, Turb. and Combust., DOI 10.1007/s10494-008-9198-3 (2009), pp. 1-24.

[9] A.R. Masri, S.S. Ibrahim, and B.J. Cadwallader, Measurements and large eddy simulation of propagating premixed flames, Exp. Therm. Fluid Sci. 30 (2006), pp. 687-702.

[10] C. Johansen and G. Ciccarelli, Visualization of the unburned gas flow field ahead of an accelerating flame in an obstructed square channel, Combust. and Flame 156 (2009), pp. 405-416

[11] T. Poinsot and D. Veynante, Theoretical and Numerical Combustion, RT Edwards, Flourtown, PA, 2001.

[12] E. Garnier, N. Adams, and P. Sagaut Large Eddy Simulation for Compressible Flows, Springer-Verlag, New York, 2009.

[13] J. Smagorinksy, General circulation experiments with the primitive equations. I. The basic experiment., Month. Weath. Rev. 91 (1963), pp. 99-164.

[14] S.B. Pope, Turbulent Flows, Cambridge University Press, New York, 2000.

[15] Fluent Inc. Fluent User Manual, Lebanon, NH: Fluent Inc., (2007).

[16] J.H. Ferziger, Subgrid-scale modeling, in Large Eddy Simulation of Complex Engineering and Geophysical Flows, B. Galperin and S.A. Orszag, Cambridge University Press, Cambridge, 1993, pp. 37-54.

[17] M. Germano, U. Piomelli, P. Moin, and W. H. Cabot, A dynamic subgrid-scale eddy-viscosity model, Phys. Fluids A 3 (1991), pp. 1760-1765.

[18] Lilly, D. K., A proposed modification of the Germano subgrid-scale closure model, Phys. Fluids A 4 (1992), pp. 633-635.

[19] Marstorp, L., Subgrid-scale modelling for large eddy simulation including scalar mixing in rotating turbulent shear flows, Technical Reports from the Royal Institute of Technology, KTH Mecahnics (2006), pp. 1-64.

[20] S.V. Patankar and D.B. Spalding, A calculation procedure for heat, mass and momentum transfer in three-dimensional parabolic flows, Int. J. Heat Mass Transfer 15 (1972), pp. 1787-1806. 
[21] B.P. Leonard, The ultimate conservative difference scheme applied to unsteady one-dimensional advection, Comp. Meth. Appl. Mech. Eng. 88 (1991), pp. 17-74.

[22] C. Johansen and G. Ciccarelli, The role of shock-flame interactions on flame acceleration in an obstacle laden channel, in 22nd ICDERS, Minsk, Belarus, 2009. 